\title{
Appreciation to Empirical Software Engineering Reviewers of 2017
}

For helping us deliver timely decisions to our authors, the Editors-in-Chief and Publisher would like to thank the following individuals that contributed reviews between November 1, 2016 and December 31, 2017. We applaud your efforts and dedication to the community.

Surafel Lemma Abebe

Bram Adams

Emil Alegroth

Nauman bin Ali

Mohammad Alshayeb

Carina Alves

Apostolos Ampatzoglou

Lefteris Angelis

Mauricio Aniche

Giulio Antoniol

John Anvik

Andrea Arcuri

Venera Arnaoudova

Paris Avgeriou

Claudia Ayala

Alberto Bacchelli

Deepika Badampudi

Ebrahim Bagheri

Hasan Bal

Sean Banerjee

Wolfgang Banzhaf

Titus Barik

Alexandre Bartel

Julian Bass

Gabriele Bavota

Andrew Begel

Jonathan Bell

Moritz Beller

Stephany Bellomo
Ayse Bener

Beatriz Bernardez

Daniel Berry

Árpad Beszedes

Cor-Paul Bezemer

David Binkley

Christian Bird

Tegawende Bissyande

Roberto Bittencourt

Alan Blackwell

Kelly Blincoe

Ivo Blohm

Remco Boer

Christopher Bogart

Markus Borg

Jürgen Börstler

Amiangshu Bosu

Goetz Botterweck

Bobby Bruce

Yuriy Brun

David Budgen

Margaret Burnett

Steven Burrows

Ray Buse

Haipeng Cai

Fabio Calefato

Joshua Campbell

Javier Canovas Izquierdo

Rafael Capilla 
Andrea Capiluppi

Fabio Casati

Marcelo Cataldo

Mariano Ceccato

Luigi Cerulo

Humberto Cervantes

Michel Chaudron

Federico Ciccozzi

Marcello Cinque

James Clause

Jane Cleland-Huang

Michael Coblenz

Michael Collard

Nelly Condori-Fernández

Steve Counsell

Kevin Crowston

Daniela Cruzes

Charlie Curtsinger

M. Daneva

Jennifer Davidson

Antinisca Di Marco

Dario Di Nucci

Massimiliano Di Penta

Oscar Dieste

Bogdan Dit

Boudewijn van Dongen

Jonathan Dorn

Robert Dyer

Sebastian Erdweg

Neil Ernst

Sandra Fabbri

John Favaro

Michael Felderer

Robert Feldt

Norman Fenton

Alessio Ferrari

Eduardo Figueiredo

Anna Filippova

Vladimir Filkov

Diego Fontdevila

Patrick Francis

Gordon Fraser

Samuel Fricker

Zak Fry

Davide Fucci

Carlo Furia

Alessio Gambi
Debin Gao

Antonio Garcia-Dominguez

Rakesh Garg

Vahid Garousi

Isabela Gasparini

Marcela Genero

Daniel German

Vincenzo Gervasi

Sepideh Ghanavati

Mohammad Gharehyazie

Rohit Gheyi

Milos Gligoric

Martin Glinz

Michael Godfrey

Jesus Gonzalez-Barahona

Sebastian Götz

Georgios Gousios

Carmine Gravino

Mark Grechanik

Alex Groce

Lars Grunske

Xiaodong Gu

Yann-Gael Gueheneuc

Latifa Guerrouj

Emitzá Guzmán

Sonia Haiduc

William Halfond

Tracy Hall

Stefan Hanenberg

Mark Harman

Rachel Harrison

Safwat Hassan

Ahmed Hassan

Edgar Hassler

Shinpei Hayashi

Steffen Herbold

Felienne Hermans

Andrea Herrmann

Abram Hindle

Reid Holmes

Helena Holmstrom Olsson

Andreas Holzinger

Shin Hong

Andre Hora

Jennifer Horkoff

Daqing Hou

LiGuo Huang 
Marianne Huchard

Ali Idri

Akinori Ihara

Jakob Iversen

Clem Izurieta

Letizia Jaccheri

Dietmar Jannach

Slinger Jansen

Yue Jia

Lingxiao Jiang

Zhen Ming (Jack) Jiang

Eirini Kalliamvakou

Yasutaka Kamei

Baris Kasikci

Christian Kästner

R. Kazman

Maria Kechagia

Jacky Keung

Hoa Khanh Dam

Foutse Khomh

Joerg Kienzle

Fitsum Meshesha Kifetew

Dongsun Kim

Jindae Kim

Miryung Kim

Mijung Kim

B. A. Kitchenham

Jacques Klein

Pavneet Singh Kochhar

Sahar Kokaly

Fábio Kon

Rainer Koschke

Samuel Kounev

Vladimir Kovalenko

Heiko Koziolek

Nicholas Kraft

Max Kramer

Sebastian Krieter

Jens Krinke

Rahul Krishna

Y. Labiche

Julia Lawall

Lucas Layman

Alina Lazar

Xuan-Bach D. Le

Claire Le Goues

Xavier Le Pallec
Seonah Lee

Jeff Lei

Philipp Leitner

Maurizio Leotta

Olaf Leßenich

Emmanuel Letier

$\mathrm{Li} \mathrm{Li}$

Mario Linares-Vásquez

Yepang Liu

David Lo

Christopher Lokan

Fan Long

Cristina Lopes

Roberto Lopez-Herrejon

Garm Lucassen

Mircea Lungu

Walid Maalej

Aravind Machiry

Ivano Malavolta

Haroon Malik

Mika Mäntylä

Andrian Marcus

Matías Martínez

Antonio Martini

Wes Masri

Rafael Matone Chanin

Andrea Mattavelli

Shane McIntosh

Collin McMillan

Phil McMinn

Sergey Mechtaev

Nenad Medvidovic

Na Meng

Tom Mens

Tim Menzies

Zhou Minghui

Leandro Minku

Mehdi Mirakhorli

Andriy Miranskyy

Andrea Mocci

Nils Moe

Akito Monden

Sandro Morasca

Laura Moreno

Sebastien Mosser

Henry Muccini

Hausi Muller 
Kıvanç Muslu

Sarah Nadi

Meiyappan Nagappan

Jaechang Nam

Ali Nassif

Maleknaz Nayebi

Hoan Nguyen

Tien Nguyen

Tung Nguyen

Nan Niu

James Noble

Nicole Novielli

Frolin Ocariza

Masao Ohira

Semih Okur

Manuel Oriol

Ipek Ozkaya

Richard Paige

Elda Paja

Fabio Palomba

Rahul Pandita

Annibale Panichella

Sebastiano Panichella

Chris Parnin

Luca Pascarella

Fabrizio Pastore

Cecile Peraire

Fayola Peters

Kai Petersen

Justyna Petke

Dorina Petriu

Dietmar Pfahl

Martin Pinzger

Macario Polo Usaola

Denys Poshyvanyk

Daryl Posnett

Pasqualina Potena

Simon Poulding

Chris Power

Rafael Prikladnicki

Laura Pullum

Fabio Queda Silva

Awais Rashid

Baishakhi Ray
Gianna Reggio

Maria Riaz

Filippo Ricca

Volker Riediger

Peter Rigby

Jurgen Rilling

Michele Risi

Romain Robbes

Gregorio Robles

Pilar Rodriguez

Simone Romano

Rasmus Ros

Julia Rubin

Ivan Ruchkin

Guenther Ruhe

Barbara Russo

Mehrdad Sabetzadeh

Zoheir Sabeur

Francesca Saglietti

Hitesh Sajnani

Guido Salvaneschi

Sreedevi Sampath

Adrián Santos

Paulo Sérgio Santos

Federica Sarro

Pete Sawyer

Giuseppe Scanniello

Sigrid Schefer-Wenzl

Kevin A. Schneider

Todd Sedano

Filippo Seracini

Alexander Serebrenik

Weiyi Shang

Bonita Sharif

David Shepherd

Emad Shihab

F. Shull

Janet Siegmund

Alberto Sillitti

Guttorm Sindre

Saurabh Sinha

Harvey Siy

Connie U. Smith

Chris Snijders 
Majid Soleimani-damaneh

Hui Song

Wei Song

Diomidis Spinellis

Paola Spoletini

Fausto Spoto

Megan Squire

Igor Steinmacher

Katie Stolee

Margaret-Anne Storey

Uta Störl

Daniel Strueber

Giancarlo Succi

Chengnian Sun

Xiaobing Sun

Richard Berntsson Svensson

Shin Hwei Tan

Antony Tang

Ewan Tempero

James Thomas

Yuan Tian

Jeff Tian

Walter Tichy

Marco Torchiano

Ayse Tosun

Erik Trainer

Guilherme Travassos

Christoph Treude

Catia Trubiani

Jason Tsay

Burak Turhan

Michael Unterkalmsteiner

Marco Tulio Valente

Jose Luis De La Vara

Bogdan Vasilescu

S. Vegas

Christopher Vendome

Colin Venters

Massimo Villari

Nair Vivek

Stefan Wagner

Robert Walker

Shaowei Wang
Tao Wang

Xiaoyin Wang

Tony Wasserman

Matthias Weidlich

Westley Weimer

Bernhard Westfechtel

Jim Whitehead

Roel Wieringa

Manuel Wimmer

W. Eric Wong

Andreas Wortmann

Franz Wotawa

Rongxin $\mathrm{Wu}$

Xin Xia

Lu Xiao

Mao Xiaoguang

Xiaoyuan Xie

Yingfei Xiong

Jifeng Xuan

Aiko Yamashita

Jinqiu Yang

Ye Yang

Annie Ying

Shin Yoo

$\mathrm{Xiao} \mathrm{Yu}$

Tingting $\mathrm{Yu}$

Tao Yue

Alexey Zagalsky

Andy Zaidman

Vadim Zaytsev

Feng Zhang

Hongyu Zhang

Pengcheng Zhang

Yiji Zhang

Yuanyuan Zhang

Yuming Zhou

Jieming Zhu

Minhaz Zibran

Franz Zieris

Thanos Zolotas

Ying Zou

Didar Zowghi 